\title{
CIRCLES IN RIEMANNIAN SYMMETRIC SPACES
}

\author{
Katsuya Mashimo* and KoJi Tojo
}

\begin{abstract}
We show that every circle in a compact Riemannian symmetic space of rank one is obtained as an orbit of a one parameter subgroup of isometries. We also show that a homogeneous space with the above property is either a Euclidean space or a Riemannian globally symmetric space of rank one.
\end{abstract}

\section{Introduction}

Let $(M, g)$ be a Riemannian manifold and $\nabla$ the Riemannian connection of $(M, g)$. An arc-length parametrized curve $c(t)$ in $(M, g)$ is called a circle if there exist a unit vector field $Y(t)$ along $c(t)$ and a positive constant $k$ such that

$$
\nabla_{\dot{c}(t)} \dot{c}(t)=k Y(t), \quad \nabla_{\dot{c}(t)} Y(t)=-k \dot{c}(t) .
$$

The constant $k$ is called the curvature of the circle. Let $\{X, Y\}$ be an arbitrary pair of mutually orthogonal unit vectors in $T_{p} M$ at a point $p \in M$ and $k$ be a positive constant. There exists a unique circle $c:(-\varepsilon, \varepsilon) \rightarrow M$ with the initial condition

$$
c(0)=p, \quad \dot{c}(0)=X, \quad\left(\nabla_{\dot{c}} \dot{c}\right)(0)=k Y
$$

for sufficiently small $\varepsilon$. It is known that in a complete Riemannian manifold every circle can be defined for $-\infty<t<\infty$.

Recently Adachi, Maeda and Udagawa [3] studied the circles in a complex projective space $P^{n}(C)$ of constant holomorphic sectional curvature. For instance, they proved that a circle in $P^{n}(C)$ is characterized by the curvature $k$ and the complex torsion. Adachi [1] studied the similar problem for a quaternion projective space and its non-compact dual. Adachi and Maeda also studied circles in complex hyperbolic space [2]. One of the purpose of this paper is to generalize their results to circles in compact Riemannian symmetric spaces of rank one.

1991 Mathematics Subject Classification. 53C40.

Key words and phrases. circle, curvature, symmetric space.

* The first author was partially supported by Grants-1n-Aid for Scientific Research (No. 07640097), The Ministry of Education, Science and Culture, Japan

Received September 5, 1997; revised November 6, 1998. 
As a part of a theorem in Maeda and Ohnita [8], every circle in a complex projective space $P^{n}(\mathbf{C})$ is obtained as an orbit of some one parameter subgroup of the full isometry group $P S U(n+1)$. In this paper we show that a homogeneous spaces $G / K$ with the property that every circle is an orbit of some one parameter subgroup of $G$ is a two point homogeneous space and vice versa.

The authors would like to thank professor S. Murakami for his useful comments and to professors R. Takagi, S. Maeda and H. Tasaki for their useful conversations and encouragement during the preparation of this paper.

\section{Orbits of one parameter subgroups}

In this section we give a necessary and sufficient condition for the existence of homogeneous circles in Riemannian homogeneous spaces.

Let $(M, g)$ be a Riemannian manifold and $c: I \rightarrow M$ be an arc-length parametrized curve in $M$. We denote by $\tau_{0}^{t}$ the parallel translation along $c$ from $c(t)$ to $c(0)$. There exists a unique curve $c^{*}: I \rightarrow T_{c(0)} M$ which satisfies

$$
\frac{d c^{*}}{d t}=\tau_{0}^{t}(\dot{c}(t)), \quad c^{*}(0)=0
$$

We call $c^{*}(t)$ the development of $c(t)$.

We call $c(t)$ a Frenet curve of osculating rank $d$ if $\left\{\dot{c}(t), \nabla_{\dot{c}} \dot{c}(t), \ldots \nabla_{c}^{d-1} \dot{c}(t)\right\}$ are linearly independent and $\left\{\dot{c}(t), \nabla_{\dot{c}} \dot{c}(t), \ldots \nabla_{c}^{d} \dot{c}(t)\right\}$ are linearly dependent at each $t \in I$. For a Frenet curve of osculating rank $d$, if we take the the Frenet frame $\left\{Y_{1}, \ldots, Y_{d}\right\}$ (the orthonormalization of $\left\{\dot{c}(t), \nabla_{\dot{c}} \dot{c}(t), \ldots \nabla_{c}^{d-1} \dot{c}(t)\right\}$ ), we have the usual Frenet-Serret's formula:

$$
\nabla_{\dot{c}}\left(Y_{1}, Y_{2}, \ldots, Y_{d}\right)=\left(Y_{1}, Y_{2}, \ldots, Y_{d}\right) A,
$$

where $A$ is a $(d, d)$-matrix

$$
A=\left(\begin{array}{cccccc}
0 & -\kappa_{1} & 0 & & & \\
\kappa_{1} & 0 & -\kappa_{2} & & & \\
0 & \kappa_{2} & 0 & \ddots & & \\
& & \ddots & \ddots & & \\
& & & & 0 & -\kappa_{d-1} \\
& & & & \kappa_{d-1} & 0
\end{array}\right)
$$

and $\kappa_{l}$ (called the $i$-th curvature function of $c(t)$ ) are positive functions. A Frenet curve of osculating rank $d$ is called a helix of order $d$ if all the curvature functions are constant. Especially, a helix of osculating rank 2 is a circle.

The following is easily verified.

LEMMA 1.1. A curve $c(t)$ is a Frenet curve of osculating rank $d$ if and only if its development $c^{*}(t)$ is a Frenet curve of osculating rank $d$. The $i$-th curvature function $\kappa_{l}$ of $c(t)$ is equal to the $i$-th curvature function $\kappa_{l}^{*}$ of $c^{*}(t)$ for any $i$ $(1 \leq i \leq d)$. 
Let $(M, g)$ be a Riemannian homogeneous space such that $M$ is the coset space of a Lie group $G$ by its compact subgroup $K$. We denote by $g$ and $f$ the Lie algebras of $G$ and $K$ respectively. Take an $\operatorname{Ad}(K)$-invariant decomposition $\mathfrak{g}=\mathfrak{f}+\mathfrak{p}$ of $\mathfrak{g}$. We will write $X_{\mathfrak{p}}$ for the $\mathfrak{p}$ component of $X \in \mathfrak{g}$. Under the canonical identification of $\mathfrak{p}$ with the tangent space $T_{o}(G / K)$ at the origin $o=\{K\}$, the restriction $\langle$,$\rangle of the Riemannian metric g$ at $o$ is an $\operatorname{Ad}(K)$ invariant scalar product on $p$.

For each $X \in \mathfrak{p}$, we define an element $\Lambda_{\mathfrak{p}}(X) \in \mathfrak{s o}(\mathfrak{p})$ by

$$
\Lambda_{\mathfrak{p}}(X)(Y)=\frac{1}{2}[X, Y]_{\mathfrak{p}}+U(X, Y), \quad X, Y \in \mathfrak{p},
$$

where

$$
\langle U(X, Y), Z\rangle=(1 / 2)\left\{\left\langle[Z, X]_{\mathfrak{p}}, Y\right\rangle+\left\langle[Z, Y]_{\mathfrak{p}}, X\right\rangle\right\}, \quad X, Y, Z \in \mathfrak{p} .
$$

Define a linear mapping $\Lambda: \mathfrak{g} \rightarrow \mathfrak{s} \mathfrak{p}(\mathfrak{p})$ by

$$
\Lambda(X)= \begin{cases}\operatorname{ad}_{X}, & X \in \mathfrak{F}, \\ \Lambda_{\mathfrak{p}}(X), & X \in \mathfrak{p} .\end{cases}
$$

We denote by $O(M)$ the bundle of all orthonomal frames on $M=G / K$. An element $X$ of $\mathfrak{g}$ generates a one parameter subgroup $\{\exp (t X)\}$ of isometries on $M$ and a one parameter subgroup of automorphisms $\left\{(\exp (t X))_{*}\right\}$ of $O(M)$. We denote by $\tilde{X}$ the vector field on $O(M)$ induced by the one parameter subgroup of automorphisms $\left\{(\exp (t X))_{*}\right\}$. The connection form $\omega$ of the connection on $O(M)$ which corresponds to the Levi-Civita connection is given by

$$
\omega(\tilde{X})=\Lambda(X), \quad X \in \mathfrak{g},
$$

(cf. [7], Vol. II, p. 201).

Now we prove the following theorem.

THEOREM 1.2. Let $G$ be a connected Lie group and $K$ be a compact subgroup. Take an element $V$ of the Lie algebra $\mathfrak{g}$ of $G$. The development $c^{*}(t)$ of a curve $c(t)=\pi(\exp (t V))$ in $M=G / K$ is given by

$$
c^{*}(t)=\sum_{j=1}^{\infty} \frac{t^{J}}{j !}(\Lambda(V))^{j-1} V_{\mathfrak{p}} .
$$

Proof. Take an orthonormal frame $u_{0}$ of $M$ at $o=e K$. By a proposition ([7], Vol. I, p. 104), the horizontal lift $v(t)$ of $c(t)$ in $O(M)$ with $v(0)=u_{0}$ is given by

$$
v(t)=(\exp (t Z))_{*}\left(u_{0}\right) \cdot e^{-t \Lambda(Z)} .
$$

Thus the parallel translation $\tau_{t}^{0}: T_{c(0)} M \rightarrow T_{c(t)} M$ is given as follows (cf. [7], Vol. I, p. 114),

$$
\tau_{t}^{0}(d \pi(Z))=(\exp (t Z))_{*}\left(e^{-t \Lambda(Z)} d \pi(Z)\right)
$$


From the definition of the development, we have

$$
(\exp (t Z))_{*}\left(e^{-t \Lambda(Z)}\left(\frac{d}{d t} c^{*}(t)\right)\right)=\dot{c}(t)=(\exp (t Z))_{*}(d \pi(Z))
$$

Thus we have

$$
\frac{d}{d t} c^{*}(t)=e^{t \Lambda(Z)} d \pi(Z)=\sum_{j=0}^{\infty} \frac{t^{\jmath}}{j !}(\Lambda(Z))^{J} d \pi(Z)
$$

Integrating both sides of the above equation, we obtain the desired result.

From Lemma 1.1 and Theorem 1.2, we easily obtain the following

THEOREM 1.3. Let $(G / K,\langle\rangle$,$) be a Riemannian homogeneous space and \mathfrak{g}, \mathfrak{f}$ and $\mathfrak{p}$ be as above. Let $\{X, Y\}$ be a pair of mutually orthogonal unit vectors in $\mathfrak{p}$ and $H$ be an element of $\mathfrak{f}$. The orbit $c(t)=\pi(\exp t(H+X))$ is a circle in $(G / K,\langle\rangle$,$) of curvature k(>0)$ with the initial condition

$$
\dot{c}(0)=X, \quad\left(\nabla_{\dot{c}} \dot{c}\right)(0)=k Y
$$

if and only if the following holds:

$$
\begin{aligned}
& \Lambda(X)(X)=[H, X]+\Lambda_{\mathfrak{p}}(X)(X)=k Y, \\
& \Lambda(X)(Y)=[H, Y]+\Lambda_{\mathfrak{p}}(X)(Y)=-k X .
\end{aligned}
$$

Corollary 1.4. Let $(G, K)$ be a Riemannian symmetric pair and $(\mathfrak{g}, \mathfrak{f})$ be the corresponding orthogonal symmetric Lie algebra. Let $\{X, Y\}$ be a pair of mutually orthogonal unit vectors in the standard complement $\mathfrak{p}$ of $\mathfrak{f}$ in $\mathfrak{g}$. The orbit $c(t)=\pi(\exp t(H+X))(H \in \mathfrak{f})$ is a circle of curvature $k(>0)$ in the Riemannian symmetric space $(G / K,\langle\rangle$,$) with the initial condition$

$$
\dot{c}(0)=X, \quad\left(\nabla_{\dot{c}} \dot{c}\right)(0)=k Y
$$

if and only if the following holds:

$$
[H, X]=k Y, \quad[H, Y]=-k X .
$$

\section{Circles in symmetric spaces of rank one}

In this section, we study circles in Riemannian symmetric space of rank one using Corollary 1.4.

THEOREM 2.1. Let $M=G / K$ be either a Euclidean space or a Riemannian symmetric space of rank one. We denote by $(\mathfrak{g}, \mathfrak{f})$ the corresponding orthogonal symmetric Lie algebra and by $\mathfrak{p}$ the orthogonal complement of $\mathfrak{f}$ in $\mathfrak{g}$. 
(i) Let $\sigma_{l}(i=1,2)$ be 2-planes in $T_{o} M$ and $\left\{X_{l}, Y_{l}\right\}$ be orthonormal basis of $\sigma_{i}$. There exists an element $k \in K$ with $k\left(X_{1}\right)=X_{2}$ and $k\left(Y_{1}\right)=Y_{2}$ if and only if the sectional curvature $K_{\sigma_{1}}$ and $K_{\sigma_{2}}$ are equal to each other.

(ii) For any pair of mutually orthonormal unit vectors $\{X, Y\}$ in $\mathfrak{p}$ there exists an element $H \in \mathfrak{f}$ which satisfies the equation (1). Namely, any circle in $M$ is expressed as an orbit of a one parameter subgroup of the full isometry group.

As a consequence we have the following congruence theorem of circles.

COROllary 2.2. Let $M$ be either a Euclidean space or a Riemannian symmetric space of rank one. Let $c_{i}(t)(i=1,2)$ be circles in $M$ ematating from $o$. We denote by $k_{l}$ the curvature of $c_{i}(t)$ and by $K_{l}$ the sectional curvature of the plane spanned by $\dot{\sigma}_{i}(0)$ and $\left(\nabla_{c_{1}} \dot{c}_{i}\right)(0)$. Two circles $c_{1}(t)$ and $c_{2}(t)$ are congruent to each other if and only if $k_{1}=k_{2}$ and $K_{1}=K_{2}$ hold.

Remark. Let $c(t)$ be a circle in a Complex projective space (resp. quaternion projective space) $M$ and $\tau$ be the complex torsion defined in [3] (resp. the structure torsion defined in [1]). The sectional curvature $K$ of the plane spanned by $\dot{c}(0)$ and $\left(\nabla_{\dot{c}} \dot{c}\right)(0)$ is given by

$$
K=k_{0}\left(1+3 \tau^{2}\right)
$$

where $k_{0}$ is the minimum of the sectional curvature of $M$.

Proof of Theorem 2.1. If $M$ is the Euclidean space the assertion (i), (ii) are trivial.

Let $c(t)$ be a circle of curvature $k(\neq 0)$ in $M=G / K$ with $c(0)=o$ and put

$$
X=\dot{c}(0), \quad Y=(1 / k) \nabla_{X} \dot{c} .
$$

If there exists $H \in \mathfrak{f}$ which satisfies (1), then the orbit $\exp (t(H+X)) \cdot o$ is the circle of curvature $k$ which satisfies (2). By the uniqueness of the circle we have $c(t)=\exp (t(H+X)) \cdot o$. Without loss of generality, we may assume that $M$ is compact and simply connected. The case in which $M$ is a sphere is trivial. The case in which $M$ is a complex projective space is a part of the result by Maeda and Ohnita [8]. So we shall prove the theorem for quaternion projective space and Cayley projective plane. The following proof for quaternion projective space is valid for sphere and complex projective space under a slight modification.

CASE $P^{n}(\boldsymbol{Q})$. Put

$$
\begin{aligned}
& G=S p(n+1)=\left\{g \in M_{n+1}(\boldsymbol{Q}): g g^{*}=I\right\} \\
& K=S p(1) \times S p(n)=\{(q, g): q \in S p(1), g \in S p(n)\},
\end{aligned}
$$

We denote by $g$ and $\mathfrak{f}$ the Lie algebras of $G$ and $K$ respectively. We denote by $m$ the orthogonal complement of $\mathfrak{f}$ in $\mathfrak{g}$ with respect to the inner product on $\mathfrak{g}$ 
defined by $\langle X, Y\rangle=-(1 / 2) \operatorname{trace}\left(X^{t} \bar{Y}\right), \quad(X, Y \in \mathfrak{g})$. Under the canonical identification

$$
\mathrm{m} \rightarrow \boldsymbol{Q}^{n} ;\left[\begin{array}{cccc}
0 & -\bar{q}_{1} & \cdots & -\bar{q}_{n} \\
q_{1} & 0 & \cdots & 0 \\
\vdots & \vdots & \ddots & \vdots \\
q_{n} & 0 & \cdots & 0
\end{array}\right] \mapsto\left[q_{1}, \ldots, q_{n}\right]
$$

the adjoint representation $K$ on $m$ is as follows;

$$
\operatorname{Ad}((q, g))\left[\ldots, q_{j}, \ldots\right]=\left[\ldots, \sum_{i=1}^{n} g_{j i} q_{i} \bar{q}, \ldots\right], \quad q \in \operatorname{Sp}(1), g=\left[g_{i j}\right] \in \operatorname{Sp}(n), q_{i} \in \boldsymbol{Q}
$$

Since $S p(n)$ acts transitively on the unit sphere in $Q^{n}$, we may assume that $X=[1,0, \ldots, 0]$. Similarly by the action of

$$
S p(n-1)=\{g \in S p(n): g X=X\}
$$

on $Q^{n}$ we may assume $Y=[u, b, 0, \ldots, 0]$ where $u+\bar{u}=0$ and $b \geq 0$. For an element $q \in S p(1)$ we put

$$
l=\left[\begin{array}{llll}
q & & & \\
& q & & \\
& & \ddots & \\
& & & q
\end{array}\right] \in K .
$$

It is easily seen that $\operatorname{Ad}(l)(X)=X$ and $\operatorname{Ad}(l)(Y)=[q u \bar{q}, b, 0, \ldots, 0]$. The group $S p(1)$ acts transitively on the unit sphere in $\boldsymbol{R} i+\boldsymbol{R} j+\boldsymbol{R} k$ by $x \rightarrow q x \bar{q}$, we may assume that

$$
X=[1,0, \ldots, 0], \quad Y=[a i, b, 0, \ldots, 0], \quad a^{2}+b^{2}=1, a \geq 0, b \geq 0 .
$$

The sectional curvature of the plane spanned by $X$ and $Y$ is equal to $4-3 b^{2}$. Thus we have (i).

If we put

$$
H=k\left[\begin{array}{ccccc}
0 & 0 & 0 & \cdots & 0 \\
0 & i a & -b & \cdots & 0 \\
0 & b & -i a & \cdots & 0 \\
\vdots & \vdots & \vdots & \ddots & \vdots \\
0 & 0 & 0 & \cdots & 0
\end{array}\right]
$$

then we have (1).

CASE $P^{2}(\mathfrak{C})$. Take an orthonormal basis $\left\{e_{0}=1, e_{1}, \ldots, e_{7}\right\}$ of the Cayley algebra $\mathbb{C}$ such that 


$$
\begin{aligned}
& e_{1} e_{2}=e_{3}, \quad e_{1} e_{4}=e_{5}, \quad e_{1} e_{6}=e_{7}, \quad e_{2} e_{5}=e_{7}, \\
& e_{2} e_{6}=e_{4}, \quad e_{3} e_{4}=e_{7}, \quad e_{3} e_{5}=e_{6} .
\end{aligned}
$$

We put

$$
\mathfrak{C}_{0}=\{u \in \mathfrak{C}: u+\bar{u}=0\}
$$

where - denotes the conjugation $\overline{\sum_{0}^{7} a_{i} e_{i}}=a_{0} e_{0}-\sum_{1}^{7} a_{i} e_{i}$.

Let $G_{i j}(0 \leq i, j \leq 7, i \neq j)$ be a skew-symmetric transformation on $\mathfrak{C}$ defined by $G_{i j}\left(e_{k}\right)=\delta_{j k} e_{i}-\delta_{i k} e_{j}(0 \leq k \leq 7)$ and define skew-symmetric transformations $F_{i j}(0 \leq i, j \leq 7, i \neq j)$ on $\mathbb{C}$ as follows;

$$
\begin{gathered}
F_{i 0}(x)=-F_{0 i}(x)=(1 / 2) e_{i} x \quad(1 \leq i \leq 7), \\
F_{i j}(x)=(1 / 2) e_{j}\left(e_{i} x\right) \quad(1 \leq i, j \leq 7, i \neq j) .
\end{gathered}
$$

Then a linear mapping $\pi: \mathfrak{s o}(8) \rightarrow \mathfrak{s o}(8)$ with

$$
\pi\left(G_{i j}\right)=F_{i j} \quad(0 \leq i, j \leq 7, i \neq j)
$$

is an automorphism on $\mathfrak{s o}(8)$ of order 2. Define a linear mapping $\kappa: S O(8) \rightarrow$ $S O(8)$ by

$$
(\kappa D)(x)=\bar{D} \bar{x}, \quad D \in S O(8), x \in \mathbb{C} .
$$

We denote also by $\kappa$ the differential mapping of $\kappa$ and put $v=\pi \circ \kappa$ : $\mathfrak{s o}(8) \rightarrow \mathfrak{s o}(8)$.

Lemma (Principle of triality [4], [5]). (i) Let $D_{1}$ be an element of $\mathfrak{s o}(8)$. There exist $D_{2}, D_{3} \in \mathfrak{s o}(8)$ which satisfy

$$
\left(D_{1} x\right) y+x\left(D_{2} y\right)=D_{3}(x y), \quad x, y \in \mathbb{C} .
$$

Furthermore $D_{2}$ and $D_{3}$ are uniquely determined from $D_{1}$. Namely

$$
D_{2}=v D_{1}, \quad D_{3}=\pi D_{1} .
$$

(ii) For any $\alpha_{1} \in S O(8)$, there exist $\alpha_{2}, \alpha_{3} \in S O(8)$ which satisfy

$$
\alpha_{1}(x) \alpha_{2}(y)=\alpha_{3}(x y), \quad x, y \in \mathfrak{C} \text {. }
$$

The following isomorphisms of Lie groups are known ([5])

$$
\begin{aligned}
& \operatorname{Spin}(8) \cong\left\{\left(\alpha_{1}, \alpha_{2}, \alpha_{3}\right) \in(S O(8))^{3}: \alpha_{1}(x) \alpha_{2}(y)=\overline{\alpha_{3}(\overline{x y})}\right\}, \\
& \operatorname{Spin}(7) \cong\left\{\alpha_{1} \in S O(8):{ }^{\exists} \alpha_{2} \in S O(7) \text { s.t. } \alpha_{1}(x) \alpha_{2}(y)=\overline{\alpha_{2}(\overline{x y})}\right\} .
\end{aligned}
$$
matrix

For $\xi=\left(\xi_{1}, \xi_{2}, \xi_{3}\right) \in \boldsymbol{R}^{3}$ and $u=\left(u_{1}, u_{2}, u_{3}\right) \in \mathbb{C}^{3}$, we denote by $X(\xi, u)$ the

$$
\left[\begin{array}{lll}
\xi_{1} & u_{3} & \bar{u}_{2} \\
\bar{u}_{3} & \xi_{2} & u_{1} \\
u_{2} & \bar{u}_{1} & \xi_{3}
\end{array}\right] .
$$


The group of automorphisms of the Jordan algebra

$$
\mathfrak{I}=\left\{X(\xi, u): \xi \in \boldsymbol{R}^{3}, u \in \mathbb{C}^{3}\right\}
$$

with the Jordan product

$$
X \circ Y=(1 / 2)(X Y+Y X), \quad X, Y \in \mathfrak{I},
$$

is the compact simple Lie group of type $\mathfrak{f}_{4}$;

$$
F_{4}=\left\{\alpha \in \operatorname{Isom}_{R}(\mathfrak{J}): \alpha(X \circ Y)=\alpha(X) \circ \alpha(Y), \quad X, Y \in \mathfrak{I}\right\} .
$$

The group $G=F_{4}$ acts transitively on the Cayley projective plane;

$$
P^{2}(\mathfrak{C})=\{X \in \mathfrak{J}: X \circ X=X, \operatorname{trace}(X)=1\} .
$$

Take 3-elements

$$
E_{1}=\left[\begin{array}{lll}
1 & 0 & 0 \\
0 & 0 & 0 \\
0 & 0 & 0
\end{array}\right], \quad E_{2}=\left[\begin{array}{lll}
0 & 0 & 0 \\
0 & 1 & 0 \\
0 & 0 & 0
\end{array}\right], \quad E_{3}=\left[\begin{array}{lll}
0 & 0 & 0 \\
0 & 0 & 0 \\
0 & 0 & 1
\end{array}\right]
$$

of $P^{2}(\mathfrak{C})$ and denote by $K_{l}(i=1,2,3)$ the isotropy subgroup in $F_{4}$ at the element $E_{l}(i=1,2,3)$. The subgroup $K_{l}(i=1,2,3)$ is isomorphic to $\operatorname{Spin}(9)$. We denote by $H$ the intersection $K_{1} \cap K_{2} \cap K_{3}$ and by $\mathfrak{h}$ the Lie algebra of $H$. We have an isomorphism $\phi: \operatorname{Spin}(8) \rightarrow H$ defined by

$$
\phi\left(\alpha_{1}, \alpha_{2}, \alpha_{3}\right)\left[\begin{array}{ccc}
\xi_{1} & u_{3} & \bar{u}_{2} \\
\bar{u}_{3} & \xi_{2} & u_{1} \\
u_{2} & \bar{u}_{1} & \xi_{3}
\end{array}\right]=\left[\begin{array}{ccc}
\xi_{1} & \alpha_{3}\left(u_{3}\right) & \overline{\alpha_{2}\left(u_{2}\right)} \\
\overline{\alpha_{3}\left(u_{3}\right)} & \xi_{2} & \alpha_{1}\left(u_{1}\right) \\
\alpha_{2}\left(u_{2}\right) & \overline{\alpha_{1}\left(u_{1}\right)} & \xi_{3}
\end{array}\right]
$$

and the isomorphism

$$
\phi_{*}:\left\{\left(D_{1}, D_{2}, D_{3}\right): D_{1} \in \mathfrak{s o}(8), D_{2}=v D_{1}, D_{3}=(k \circ \pi) D_{1}\right\} \rightarrow \mathfrak{h}
$$

defined by

$$
\phi_{*}\left(D_{1}, D_{2}, D_{3}\right)\left[\begin{array}{ccc}
\xi_{1} & u_{3} & \bar{u}_{2} \\
\bar{u}_{3} & \xi_{2} & u_{1} \\
u_{2} & \bar{u}_{1} & \xi_{3}
\end{array}\right]=\left[\begin{array}{ccc}
0 & D_{3} u_{3} & \overline{D_{2} u_{2}} \\
\overline{D_{3} u_{3}} & 0 & D_{1} u_{1} \\
D_{2} u_{2} & \overline{D_{1} u_{1}} & 0
\end{array}\right]
$$

Remember that $k \circ \pi=v^{2}$. We put

$$
\mathfrak{M}^{-}=\left\{\left[\begin{array}{ccc}
0 & u_{3} & -\bar{u}_{2} \\
-\bar{u}_{3} & 0 & u_{1} \\
u_{2} & -\bar{u}_{1} & 0
\end{array}\right]: u_{1}, u_{2}, u_{3} \in \mathbb{C}\right\}
$$

and for each $A \in \mathfrak{M}^{-}$define a homomorphism $\tilde{A}: \mathfrak{J} \rightarrow \mathfrak{J}$ by

$$
\tilde{A} X=[A, X] \quad \forall X \in \mathfrak{J} .
$$


The Lie algebra $\mathfrak{f}_{4}$ is decomposed as follows

$$
\mathfrak{f}_{4}=\mathfrak{h} \oplus\left\{\tilde{A}: A \in \mathfrak{M}^{-}\right\} .
$$

For a Cayley number $x \in \mathbb{C}$ we put

$$
A_{1}(x)=\left[\begin{array}{ccc}
0 & 0 & 0 \\
0 & 0 & x \\
0 & -\bar{x} & 0
\end{array}\right], \quad A_{2}(x)\left[\begin{array}{ccc}
0 & 0 & -\bar{x} \\
0 & 0 & 0 \\
x & 0 & 0
\end{array}\right], \quad A_{3}(x)=\left[\begin{array}{ccc}
0 & x & 0 \\
-\bar{x} & 0 & 0 \\
0 & 0 & 0
\end{array}\right]
$$

and write $\tilde{A}_{i}(x)$ for $\widetilde{A_{i}(x)}(1 \leq i \leq 3)$. The Killing form $B$ on $\mathfrak{f}_{4}$ is given by

$$
B(X, X)=-9 \operatorname{trace}\left(D_{1}{ }^{t} D_{1}\right)-72 \sum_{i=1}^{3} u_{i} \bar{u}_{i}
$$

where $X=\phi_{*}\left(D_{1}, D_{2}, D_{3}\right)+\sum_{t=1}^{3} \tilde{A}_{i}\left(u_{i}\right) \in \mathfrak{f}_{4}$. We denote by $\langle$,$\rangle the invariant$ Riemannian metric on the Cayley projective plane induced from the inner product $-(1 / 72) B$ on $\mathfrak{f}_{4}$. We have the following relations;

$$
\left[\tilde{A}_{i}(a), \tilde{A}_{l+1}(b)\right]=-\tilde{A}_{l+2}(\overline{a b}),\left[\phi_{*}\left(D_{1}, D_{2}, D_{3}\right), \tilde{A}_{i}(a)\right]=\tilde{A}_{i}\left(D_{i} a\right)
$$

where indices in the first relation are considered modulo 3 . The tangent space at $o=E_{1}$ of the Cayley projective plane is identified with

$$
T_{o}\left(P^{2}(\mathfrak{C})\right)=\left\{\tilde{A}_{2}(x)+\tilde{A}_{3}(y): x, y \in \mathfrak{C}\right\} .
$$

Since the action of $K=K_{1} \cong \operatorname{Spin}(9)$ on the unit sphere $S^{15}$ in $T_{o}\left(P^{2}(\mathfrak{C})\right)$ is transitive we may assume that $X=\tilde{A}_{2}\left(e_{0}\right)$. The isotropy subgroup at $\tilde{A}_{2}\left(e_{0}\right)$ of the action of $\operatorname{Spin}(9)$ on $S^{15}$ is isomorphic to $\operatorname{Spin}(7)$. Under the isotropy representation of $\operatorname{Spin}(7)$, the tangent space $T_{X} S^{15}$ is decomposed into two irreducible components;

$$
\left\{\tilde{A}_{2}(x): x \in \mathfrak{C}_{0}\right\} \oplus\left\{\tilde{A}_{3}(y): y \in \mathbb{C}\right\} .
$$

Since the action of $\operatorname{Spin}(7)$ on $\left\{\tilde{A}_{3}(y): y \in \mathbb{C}\right\}$ is transitive we may assume that $Y=\tilde{A}_{2}(a)+\tilde{A}_{3}\left(b e_{0}\right), \mathfrak{C}_{0} \ni a, b \geq 0$. Consider the subgroup

$$
H^{\prime}=\left\{k \in K_{1}: k\left(\tilde{A}_{2}\left(e_{0}\right)\right)=\tilde{A}_{2}\left(e_{0}\right), \quad k\left(\tilde{A}_{3}\left(e_{0}\right)\right)=\tilde{A}_{3}\left(e_{0}\right)\right\} .
$$

Since $H^{\prime}$ is isomorphic to the exceptional compact simple Lie group $G_{2}$ and it acts transitively on the unit sphere $S^{6}$ in $\left\{\tilde{A}_{2}(x): x \in \mathfrak{C}_{0}\right\}$, we may assume that $Y=\tilde{A}_{2}\left(a e_{1}\right)+\tilde{A}_{3}\left(b e_{0}\right) \quad\left(a^{2}+b^{2}=1, a \geq 0, b \geq 0\right)$. For each $u \in \mathfrak{C}$ we define three linear endomorphisms $L_{u}, R_{u}$ and $T_{u}$ on $\mathbb{C}$ by

$$
L_{u}(x)=u x, \quad R_{u}(x)=x u, \quad T_{u}(x)=u x+x u .
$$

If $u \in \mathfrak{C}_{0}$, then $L_{u}, R_{u}$ and $T_{u}$ are elements of $\mathfrak{s o}(8)$. By a direct calculation, we have

$$
[X, Y]=-\tilde{A}_{1}\left(b e_{0}\right)+\phi_{*}\left(R_{-2 a e_{1}}, T_{2 a e_{1}}, L_{-2 a e_{1}}\right)
$$


and the sectional curvature of the plane spanned by $X$ and $Y$ is equal to $4-3 b^{2}$. Thus we have (i).

Put $\alpha=a e_{1}$ and

$$
D_{1}=-T_{\alpha}, \quad D_{2}=v D_{1}=L_{\alpha}, \quad D_{3}=v^{2} D_{1}=R_{\alpha} .
$$

For $H=k\left(\phi_{*}\left(D_{1}, D_{2}, D_{3}\right)-\tilde{A}_{1}\left(b e_{0}\right)\right) \in \mathfrak{l}$, we have from (3)

$$
\begin{aligned}
{[H, X] } & =k\left(\tilde{A}_{2}\left(D_{2}\left(e_{0}\right)\right)+\tilde{A}_{3}\left(b e_{0}\right)\right)=k Y \\
{[H, Y] } & =k\left(\tilde{A}_{2}\left(D_{2}\left(a e_{1}\right)+b\left(-b e_{0}\right)\right)+\tilde{A}_{3}\left(D_{3}\left(b e_{0}\right)-a e_{1}\left(b e_{0}\right)\right)\right) \\
& =-k X .
\end{aligned}
$$

Remark. The maximum of the sectional curvature of $\left(P^{2}(\mathfrak{C}),\langle\rangle,\right)$ is equal to 4 .

Proposition 2.3. Let $M$ be the complex projective space $P^{n}(C)$, the quaternion projective space $P^{n}(Q)$ or the Cayley projective plane $P^{2}(\mathfrak{C})$ equipped with a canonical invariant metric. For any circle $c(t)$ of curvature $k(\neq 0)$, there exists a totally geodesically embedded complex projective space $P^{2}(C)$ which contains the circle.

Proof. The case that $M$ is the complex projective space or the quaternion projective space is trivial. So we shall prove the Proposition for the case of Cayley projective plane.

We retain the same notation as in Theorem 2.2. If we put

$$
S_{i}=\tilde{A}_{i}\left(e_{0}\right), \quad T_{l}=\tilde{A}_{i}\left(e_{1}\right) \quad(1 \leq i \leq 3)
$$

we have

$$
\left[S_{i}, S_{i+1}\right]=-\left[T_{l}, T_{l+1}\right]=-S_{i+2}, \quad\left[S_{i}, T_{l+1}\right]=\left[T_{l}, S_{i+1}\right]=T_{l+2}
$$

where the indices are considered as modulo 3. Since $\left\{2 \phi_{*}\left(G_{i j}, v G_{i j}, v^{2} G_{i j}\right): 0 \leq\right.$ $i<j \leq 7\}$ is an orthonormal basis of $\mathfrak{h}$, it is easily verified that

$$
\begin{aligned}
& {\left[S_{2}, T_{2}\right]=4 \phi_{*}\left(v^{2} G_{10}, G_{10}, v G_{10}\right),} \\
& {\left[S_{3}, T_{3}\right]=4 \phi_{*}\left(v G_{10}, v^{2} G_{10}, G_{10}\right) .}
\end{aligned}
$$

The subspace spanned by $\left\{G_{01}, G_{23}, G_{45}, G_{67}\right\}$ in $\mathfrak{s v}(8)$ is invariant under the automorphism $v$. Thus the subspace $\mathfrak{s}$ spanned by $\left\{S_{2}, S_{3}, T_{2}, T_{3}\right\}$ is a Lie triple system and $[\mathfrak{s ,}, \mathfrak{s}]+\mathfrak{s}$ is isomorphic to $\mathfrak{s u}(3)$. Let $U$ be the subgroup of $F_{4}$ of which Lie algebra is $[\mathfrak{s}, \mathfrak{s}]+\mathfrak{s}$ and $N$ be the totally geodesic submanifold generated by the Lie triple system $\mathfrak{s}$. It is clear that $N$ is diffeomorphic to $P^{2}(C)$ and contains the circle $\{c(t)\}$. 
THEOREM 2.4. Let $M$ be a complex projective spaces $P^{n}(C)$, a quaternion projective space $P^{n}(Q)$ or a Cayley projective plane $P^{2}(\mathfrak{C})$. Equip $M$ with the canonical invariant metric such that the maximum of the sectional curvature is equal to 4 . Let $c(t)$ be a circle with curvature $k(\neq 0)$ in $M$. We denote by $K$ the sectional curvature of the tangent 2-plane spanned by $\dot{c}(0)$ and $\left(\nabla_{\dot{c}} \dot{c}\right)(0)$.

Case $K=4$ : The circle $c(t)$ is a simple closed curve with prime period $2 \pi / \sqrt{k^{2}+4}$.

Case $K=1$ : The circle $c(t)$ is a simple closed curve with prime period $2 \pi / \sqrt{k^{2}+1}$.

Case $K \neq 1,4$ : Suppose that $\alpha_{1}, \alpha_{2}$ and $\alpha_{3}\left(\alpha_{1}<\alpha_{2}<\alpha_{3}\right)$ be nonzero solutions of the equation;

$$
\lambda^{3}-\left(k^{2}+1\right) \lambda-k \sqrt{(K-1) / 3}=0 .
$$

Then the circle $c(t)$ is closed if and only if one of the three ratios $\alpha_{1} / \alpha_{2}, \alpha_{2} / \alpha_{3}$ and $\alpha_{3} / \alpha_{1}$ is rational. Moreover, the prime period of $c(t)$ is the least common multiple of $2 \pi /\left(\alpha_{2}-\alpha_{3}\right)$ and $2 \pi /\left(\alpha_{3}-\alpha_{1}\right)$.

Adachi, Maeda and Udagawa [3] proved the theorem for the case that $M$ is the complex projective space. From Proposition 2.3, the theorem is a direct consequence of their theorem. We shall give another proof.

Proof. For any circle $c(t)$ of curvature $k$ in $M$, we can take a totally geodesically embedded submanifold $P^{2}(C)$ which contains the circle. Thus we assume that $M=P^{2}(C)$.

Take any circle $c(t)$ in $P^{2}(C)$. Without loss of generality we may assume that $c(0)$ is the origin $[1,0,0]$. There exist real numbers $a, b \in \boldsymbol{R}\left(a^{2}+b^{2}=1\right)$ such that $c(t)$ is the orbit of the one parameter subgroup $\exp (t(k H+X))$ for

$$
H=\left[\begin{array}{ccc}
0 & 0 & 0 \\
0 & i a & -b \\
0 & b & -i a
\end{array}\right], \quad X=\left[\begin{array}{ccc}
0 & -1 & 0 \\
1 & 0 & 0 \\
0 & 0 & 0
\end{array}\right]
$$

CASE $a=0$ or 1 . In this case there exists a totally geodesically embedded submanifold $P^{2}(\boldsymbol{R})$ or $P^{1}(\boldsymbol{C})=S^{2}$ which contains the circle. Thus the assertion of the theorem is trivial.

CASE $a b \neq 0$. We denote by $i \alpha_{1}, i \alpha_{2}$ and $i \alpha_{3}$ the eigenvalues of $k H+X$, namely $\alpha_{1}, \alpha_{2}$ and $\alpha_{3}$ are the solutions of the characteristic equation;

$$
|(k H+X)-i \lambda I|=i\left(\lambda^{3}-\lambda\left(k^{2}+1\right)-a k\right) .
$$

Since the sectional curvature $K$ of the 2-plane spanned by $X$ and $[H, X]$ is equal to $3 a^{2}+1$ we have $a=\sqrt{(K-1) / 3}$. 
Remember that the solutions of the above equation are mutually distinct. Take a unitary matrix $U$ which diagonalize the matrix $V=k H+X$;

$$
U V U^{-1}=\left[\begin{array}{ccc}
i \alpha_{1} & 0 & 0 \\
0 & i \alpha_{2} & 0 \\
0 & 0 & i \alpha_{3}
\end{array}\right]
$$

By an easy calculation we can show that none of the entry of the eigenvector of $V$ (accordingly none of the entry of $U$ nor $U^{-1}$ ) is zero. Thus none of the entry of the matrix

$$
Z=U\left[\begin{array}{lll}
1 & 0 & 0 \\
0 & 0 & 0 \\
0 & 0 & 0
\end{array}\right] U^{-1}
$$

is zero.

We denote by $\operatorname{Sym}_{3}(C)$ the set of all $3 \times 3$ complex symmetric matrices and by $E_{1}$ the element of $\operatorname{Sym}_{3}(C)$ whose $(1,1)$ entry is equal to 1 and other entries are zero. The mapping $S U(3) \rightarrow \operatorname{Sym}_{3}(C) ; g \mapsto g E_{1} g^{-1}$ induces an embedding $\imath: P^{2}(C) \rightarrow \operatorname{Sym}_{3}(C)$. The image of the circle $c(t)=\exp (t V) \cdot o$ is

$$
\imath(c(t))=U^{-1} D_{t} Z D_{-t} U
$$

where

$$
D_{t}=\left[\begin{array}{ccc}
e^{i t \alpha_{1}} & 0 & 0 \\
0 & e^{i t \alpha_{2}} & 0 \\
0 & 0 & e^{i t \alpha_{3}}
\end{array}\right]
$$

If we assume that $c(0)=c(t)$ then $l_{*}(\dot{c}(0))=l_{*}(\dot{c}(t))$. Namely $c(t)$ is a simple curve. It is easily seen that the condition $c(0)=c(t)$ is equivalent to the condition

$$
\left(\alpha_{i}-\alpha_{j}\right) t \in 2 \pi Z \quad(1 \leq i, j \leq 3) .
$$

The assertion of the theorem is an immediate consequence.

\section{Circles in homogeneous spaces}

We devote this section to a proof of the following theorem.

THEOREM 3.1. Let $(M, g)$ be a Riemannian homogeneous space and $k$ be a positive constant. If all the circles of curvature $k$ are orbits of one parameter subgroups in the isometry group of $(M, g)$ then $(M, g)$ is either a Euclidean space or a Riemannian globally symmetric space of rank one.

Proof. The 'only if' part of the theorem is the assertion of Theorem 2.1. In the following we prove the 'if' part. 
Suppose that all the circles in $(G / K,\langle\rangle$,$) are orbits of one parameter$ subgroups in $G$. Let $\{X, Y\}$ be an arbitrary pair of mutually orthogonal unit vectors in $\mathfrak{p}$. Take two circles $c_{1}$ and $c_{2}$ emanating from $0=e K$ which satisfy $\dot{c}_{1}(0)=\dot{c}_{2}(0)=X$ and $\left(\nabla_{c_{1}} \dot{c}_{1}\right)(0)=-\left(\nabla_{c_{2}} \dot{c}_{2}\right)(0)=k Y$. From Theorem 1.3, there exists elements $H_{1}$ and $H_{2}$ in $\mathfrak{f}$ such that the following hold.

$$
\begin{aligned}
& {\left[H_{1}, X\right]+\Lambda_{\mathfrak{p}}(X)(X)=k Y,} \\
& {\left[H_{2}, X\right]+\Lambda_{\mathfrak{p}}(X)(X)=k(-Y) .}
\end{aligned}
$$

Thus for $H=(1 / 2 k)\left(H_{1}-H_{2}\right)$, we have

$$
[H, X]=Y \text {. }
$$

To prove the theorem we shall show that the linear isotropy $\operatorname{group} \operatorname{Ad}(K)$ acts transitively on the unit sphere $S(\mathfrak{p})$ in $\mathfrak{p}([6, \mathrm{p} .535])$.

Consider the mapping $\phi: K \rightarrow S(\mathfrak{p})$ by $\phi(k)=\operatorname{Ad}(k) \cdot X$. Since $K$ is compact, $\phi(K)$ is closed in $S(\mathfrak{p})$.

For each $k_{0} \in K$, identify the tangent space $T_{\phi\left(k_{0}\right)} S(\mathfrak{p})$ with $\left\{\operatorname{Ad}\left(k_{0}\right) \cdot Y\right.$; $Y \in \mathfrak{p}, Y \perp X\}$. The differential mapping of $\phi$, given by

$$
(d \phi)_{k_{0}}(H)=\operatorname{Ad}\left(k_{0}\right) \cdot[H, X], \quad H \in \mathfrak{f},
$$

is surjective for any $k_{0} \in K$ by (4). Therefore, from the implicit function theorem, we can see that $\operatorname{Ad}(K) \cdot X$ is open in $S(\mathfrak{p})$. Consequently we have $\operatorname{Ad}(K) \cdot X=S(\mathfrak{p})$.

Considering the proof of Theorem 3.1, we have the following.

COROLlaRY 3.2. Let $(M, g)$ be a Riemannian homogeneous space and $p \in M$. Then there exists a vector $X$ in $T_{p} M$ such that all the circles $c(t)$ in $(M, g)$ with $\dot{c}(0)=X$ are orbits of one parameter subgroups in the isometry group of $(M, g)$ if and only if $(M, g)$ is two-point homogeneous.

\section{REFERENCES}

[1] T. ADACHI, Circles on quaternionic space forms, J. Math. Soc. Japan, 48 (1996), 205-227

[2] T. ADACHI AND S. MAEDA, Global behaviour of circles in a complex hyperbolic space, Tsukuba J. Math., 20 (1996), 29-42.

[3] T. Adachi, S. Maeda and S. Udagawa, Circles in complex projective space, Osaka J. Math., 32 (1995), 709-719.

[4] H. Freudenthal, Oktaven, Ausnahmegruppen und Oktavengeometre, Geom. Dedicata, 19 (1985), 7-63.

[5] R. Harvey, Spinors and Calibrations, Academic Press, 1990.

[6] S. Helgason, Differential Geometry, Lie Groups, and Symmetric Spaces, Academic Press, 1978.

[7] S. Kobayashi and K. Nomizu, Foundations of Differential Geometry I, II, Interscience, 1963. 
[8] S. Maeda AND Y OhNita, Helical geodesic immersions into complex space froms, Geom. Dedicata, 30 (1989), 93-114.

Department of Mathematics

Tokyo University of Agriculture and TeChNology

FUCHU, TOKYO 183-0054

JAPAN

DepartMent OF Mathematics

Chiba Institute of TeChNology

Shibazono, Narashino, Chiba 275-0023

JAPAN 